\title{
Optimization of Marine Medium Speed Diesel Engine Performance based on Multi-Injector System
}

\author{
Dai Liu ${ }^{1 \mathrm{a}}$, Yingzhu Guo ${ }^{1 \mathrm{~b}}$, Long Liu ${ }^{1 \mathrm{c} *}$, Qian $\mathrm{Xia}^{2 \mathrm{~d}}$ and Yong Gui² \\ ${ }^{1}$ Harbin Engineering University,College of Power and Energy Engineering, Nangang district, Harbin, China \\ ${ }^{2}$ China Shipbuilding Power Engineering Institute Co Ltd, Port industrial zone, Pudong new area, Shanghai, China
}

\begin{abstract}
Multi-injector system is potential to improve thermal efficiency and NOx emission of diesel engine at the same time. In order to optimize the combustion and emission of Marine medium speed diesel engine, the engine combustion with a multi-injector system is simulated and analyzed by CFD software Converge. In this research, two injectors are installed at the side of the cylinder head while the central injector is maintained. Various injection directions of side injectors and injection strategies of multi-injector system are simulated to optimize the fuel spray and combustion. The analysis results show that the spray angle of the side injector plays a key role for effective thermal efficiency improvement, since complex spray jet-jet interaction and spray impingement may deteriorate the combustion if the arrangement of spray angle was not set properly. Once the fuel injection direction has been optimized, the fuel ratio of the three injectors is optimized and improved the effective thermal efficiency with lower NOx emission. The results show that the two side injectors could increase the fuel injection rate into the cylinder, leading to high brake power and consequently increased the thermal efficiency by $1.26 \%$ and decreased the NOx emission by $16 \%$ for the best optimization.
\end{abstract}

\section{Introduction}

With the exhaustion of petroleum fuel and increasingly severe environmental pollution, more stringent requirements on the economy and emission of Marine diesel engines has been proposed. The large bore diameter (normally more than $250 \mathrm{~mm}$ ) and long injection duration (can reach $5 \mathrm{~ms}$ for high load) of medium-speeds diesel engine leads to increasing of near wall combustion and long combustion duration. Both of them would result in a decreasing of the thermal efficiency.

To solve this problem, a novel fuel system with two or more injectors per cylinder is proposed. The novel multiinjector system is expected to provide three advantages in combustion: Firstly, shorten the fuel injection duration. More fuel could be injected into the cylinder to reduce the combustion duration and improve the thermal efficiency. Secondly, the mixture is facilitated by a continuous flow of air upstream of the spray. Through numerical and experimental results, Koci et al. ${ }^{[1]}$ found that the external air-assisted fuels spray may reduce the local equivalence ratio by enhancing the fuel/air mixing process. Thirdly, the heat loss of the wall can be reduced since the fuel injected from the side injectors is concentrated in the center of the cylinder and consequently reduced the impact of flame near the cylinder wall. Several studies have been conducted on diesel engines with multiple injectors, such as the PREDIC ${ }^{[2]}$ and MULDIC ${ }^{[3]}$ concepts proposed in the Premix compression ignition (PCCI) combustion control study. Researchers have confirmed that the use of multiple injectors can reduce heat loss ${ }^{[4,5]}$.

Noboru Uchida et al. ${ }^{[6]}$ carried out numerical analysis and tests to investigate the combustion and emission characteristics of diesel engines by adopting multi-injector and newly designed cavity single cylinder engine. Their results indicated that friction loss, heat loss and NOx emissions can be reduced while maintaining the indicated thermal efficiency by independently controlling the injection timing of each injector. In addition, the improved nozzle with large flow coefficient and reduced holes is used in the side injector to generate the swirl spray and improved the braking thermal efficiency significantly. ${ }^{[7]}$. Takeda $\mathrm{Y}$ and et al ${ }^{[8]}$ installed three injectors in a traditional single cylinder diesel engine. One injector was installed vertically in the center of the cylinder, the other two injectors was installed on the side of the cylinder. through the experiments, they confirmed several optimization settings for the fuel quantity and injection timing of each injector. Both particle emission and fuel consumption were improved with remained low NOx levels. Gustav ${ }^{[9]}$ also carried out experiments on a heavyduty engine with multi-injector system. He further investigated the spray and combustion behavior through optical engine, and compared the flame structures of single-injector and multiinjector system. Compared with the single injector system,

\footnotetext{
adailiu@hrbeu.edu.cn_byz12072020@126.com * Corresponding author: c liulong@hrbeu.edu.cn d xq@espi.net.cn

e gyong@cspi.net.cn
} 
the high temperature zone is located further away from the cylinder boundaries in the cases of three-injector system, which leads to the reduction of heat loss and improvement of braking thermal efficiency.

The diesel engines in the above examples are all vehicle engines, indicating that the novel fuel system can reduce or maintain emissions and improve thermal efficiency at the same time for the diesel engines of smaller size .The combustion chamber of marine diesel engine is much larger than that of the vehicle diesel engine, which is an advantage for multiple injectors arrangement and fuel/air mixing under the condition of complex fuel jet distribution in the cylinder. Therefore, the novel fuel system is applied in a marine diesel engine through a validated 3D model. Two injectors with 3 nozzle holes are set on the side of the cylinder head, while the original injector is maintained. The spray orientations of each hole in the side injector were simulated to ensure the best thermal efficiency. However, the optimized nozzle structures of side injectors are not able to increase the thermal efficiency if the injection strategy was not properly optimized. After further combined with proper injection strategy, the thermal efficiency was increased with NOx emission decreased finally.

\section{Simulation model, model validation and work scheduling}

\subsection{Simulation model}

In this study, CFD software CONVERGE was applied to conduct simulation modeling on a marine medium-speed diesel engine, and the configuration of the diesel engine was shown in Table 1 . The basic mesh size is $20.0 \mathrm{~mm}$, applying adaptive mesh refinement for velocity and temperature. The

Table 1 Engine specifications.

\begin{tabular}{|l|l|}
\multicolumn{2}{|c|}{ Table 1 Engine specifications. } \\
\begin{tabular}{|l|l|} 
Base Engine Configuration & Single cylinder $/ 4$ stroke \\
Displacement & $550 \mathrm{~mm}$ \\
\hline Stroke & $390 \mathrm{~mm}$ \\
\hline Bore & $1200 \mathrm{~mm}$ \\
\hline Connecting Rod & $17.5: 1$ \\
\hline Fuel Injection System & Common rail \\
\hline Center nozzle orifice & $\phi 0.63 \mathrm{~mm} \times 12(0.1257 \mathrm{~m} 3 / \mathrm{min})$ \\
\hline Side nozzle orifice & $\phi 0.63 \mathrm{~mm} \times 3(0.0314 \mathrm{~m} 3 / \mathrm{min}) /$ \\
& $\phi 0.8 \mathrm{~mm} \times 3(0.0507 \mathrm{~m} 3 / \mathrm{min})$ \\
\hline Maximum injection pressure & $1500 \mathrm{Mpa}$ \\
\hline
\end{tabular}
\end{tabular}

subdividing criteria are differences of $1.0 \mathrm{~m} / \mathrm{s}$ and $2.5 \mathrm{~K}$ in the adjoining meshes, respectively. The area where the fuel injector located is encrypted layer by layer along the injection direction of the fuel spray. The minimum grid size are 0.625

$\mathrm{mm}$. The $\mathrm{O}$ 'Rourke model was used for wall heat transfer calculation, and the sub-models applied for simulation engineering were shown in Table 2.

Table 2. Sub-models used by CONVERGE.

\begin{tabular}{|l|l|}
\hline Phenomenon & Model \\
\hline Turbulence & RNG k- $-\varepsilon$ \\
\hline Combustion & $\begin{array}{l}\text { Characteristic time combustion } \\
\text { (CTC) }\end{array}$ \\
\hline
\end{tabular}

\begin{tabular}{|l|l|}
\hline Auto ignition & Shell \\
\hline Spray breakup & KH-RT \\
\hline
\end{tabular}

\subsection{Model validation}

In the engine model, the simulation only covers the combustion process from inlet valve closing to exhaust valve opening. In order to ensure the accuracy of the simulation results, the spray and combustion sub-models were calibrated according to the experimental data and the calibration results are shown in figure 1 and 2, respectively. The experimental results of spray is from literature ${ }^{[10]}$ and the experimental data of engine combustion are provided by manufacturer. It is obvious that the simulated fuel spray agreed with experimental data well. Then, parameters of the spray sub-model are maintained for the combustion calibration. The comparison of simulated cylinder pressure curve and experimental data under three loads is shown in FIG. 2. The curves agree well and the model can be used for further research.

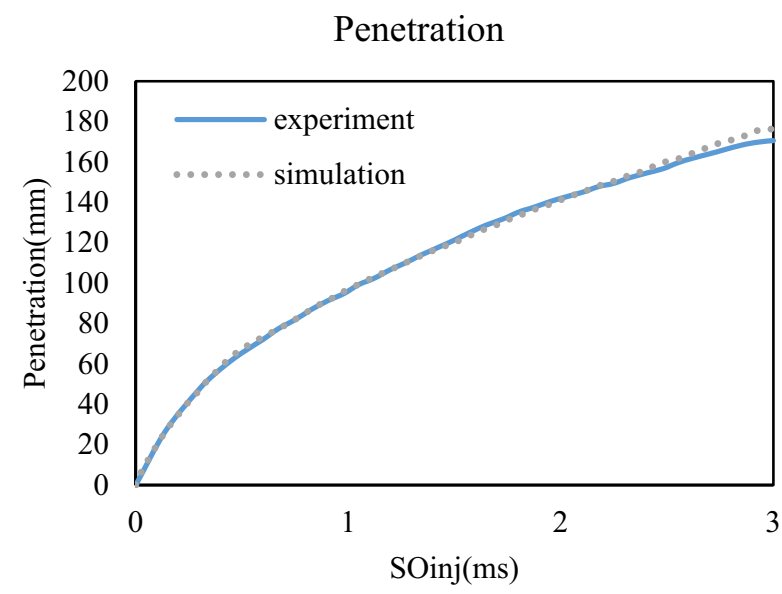

Figure 1 shows the overall data comparison In-cylinder Pressure

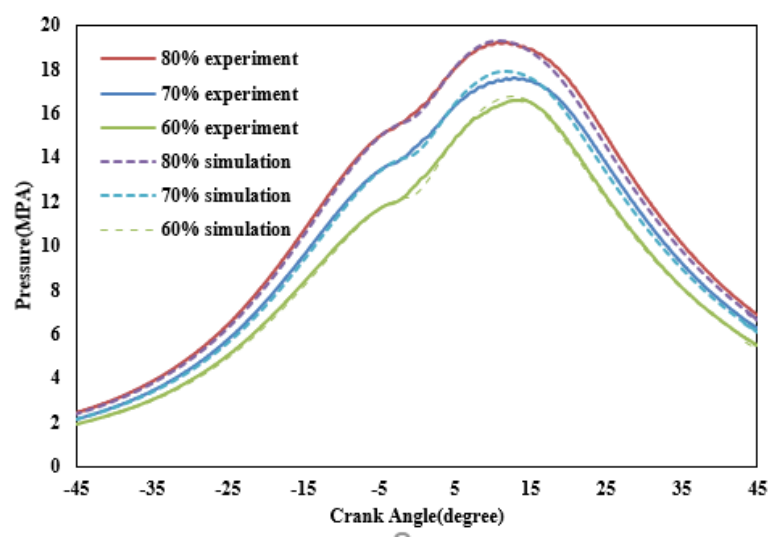

Figure. 2 Comparison of cylinder pressure between simulation and experiment

\subsection{Work scheduling}

In order to compare the combustion characteristics and thermal efficiency after replacing the fuel injection system, a series of simulation were carried out (Table 2). The 
injector structure and arrangement in the simulation were shown in Figure 3. The start of injection was set at -6CAD for each injector and fuel quantity was set as $4: 3: 3$, which means that the central injector provides $40 \%$ of fuel quantity and the side injectors provide $30 \%$ for each. The position variations of the side injector are determined through the observation of fuel spray distribution and interaction in the combustion chamber before simulation. If there are obvious increased fuel interaction and wall impingement, the positions and nozzle orientations at this condition were set as boundary position. Therefore, the variations of nozzle orientations can be limited to a smaller range for simulation. Considering the cylinder head structure and the position of the valves, the position of the side injector is setting $175 \mathrm{~mm}$ away from the center of the cylinder head. The side injector is installed at an inclined Angle of $25^{\circ}$ from the axis and its nozzle orientations is varied as shown in table 3 for optimization.

Table 3, Simulation cases matrix

\begin{tabular}{|c|c|c|c|}
\hline$\alpha_{1}(\operatorname{deg})$ & $\alpha_{2}(\operatorname{deg})$ & $\beta_{1}(\operatorname{deg})$ & $\beta_{2}(\operatorname{deg})$ \\
& & & \\
\hline$-10 / 0 / 8$ & 20 & 12.5 & 7 \\
\hline-10 & $19 / 20 / 23$ & 12.5 & 7 \\
\hline-10 & 20 & 12.5 & $5 / 7 / 9$ \\
\hline-10 & 20 & $10.5 / 12.5 / 14.5$ & 7 \\
\hline
\end{tabular}
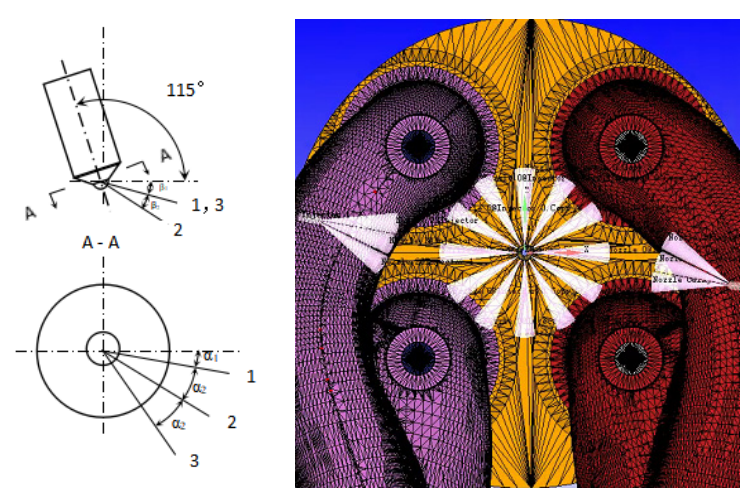

Figure. 3 Schematic diagram of simulation related parameters

\section{Results and Discussion}

The spray direction and fuel quantity ratio determine the spray distribution and interaction, which affects the fuel/air mixing and combustion process. Various cross section angle, longitudinal section angle and fuel quantity ratio were simulated.

\subsection{Influence of cross section angle}

The effect of $\alpha 1$ on combustion and performance is shown in Figure 4, 5 and 6. Both of the heat loss and effective thermal efficiency are reduced when $\alpha 1$ reduced from 8 degree to -10degree. Although the heat loss is reduced obviously, the overall effective thermal efficiency of the multi-injector case is still lower than that of the original engine, which is due to the increasing of exhaust loss. The size of the side injector is limited by the space of cylinder head (valves and coolant channel). Therefore, the highest injection quantity of the selected side injector is only $1 / 4$ of the main injector, results in a longer injection duration and consequently a longer combustion duration with higher exhaust loss. However, it is still worthy to analyze the distribution of high temperature area in the combustion chamber, which is the main influencing factor for heat loss. Figure 5 reflects the effect of $\alpha 1$ on the temperature distribution in the combustion chamber (the sectional plane is $20 \mathrm{~mm}$ away from the cylinder head). All multiinjector cases show a tendency of increased combustion zone in the center of cylinder. The change of $\alpha 1$ means that the overall injection direction changes, which affects the distribution of the fuel combustion in the cylinder. As $\alpha 1$ reduced, of the fuel penetration of side injector to the center of the combustion chamber is reduced due to the interaction of fuel jet from the central injector, and the high temperature region of the central part showed a tendency to be expanded. In addition, the heat release rate at the later stage of combustion (around 20CAD ATDC) tends to increase as $\alpha 1$

increases (shown in figure 6), which can be explained by the reduced interaction of fuel jet from different injectors shown

in figure 5. Overall, the $\alpha 1$ indicated an obvious influence in spray and combustion distribution, a slightly larger angle can reduce the fuel jet-jet interaction and promote the combustion and thermal efficiency, even though there is a slight increase in heat loss (around $0.2 \%$ ).

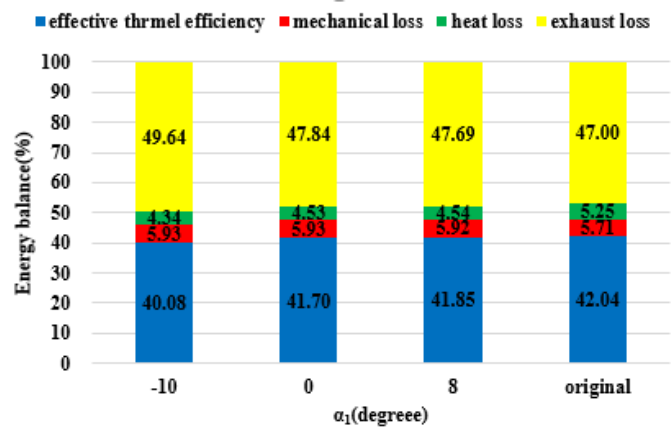

Figure. 4 Influence of $\alpha_{1}$ on energy balance

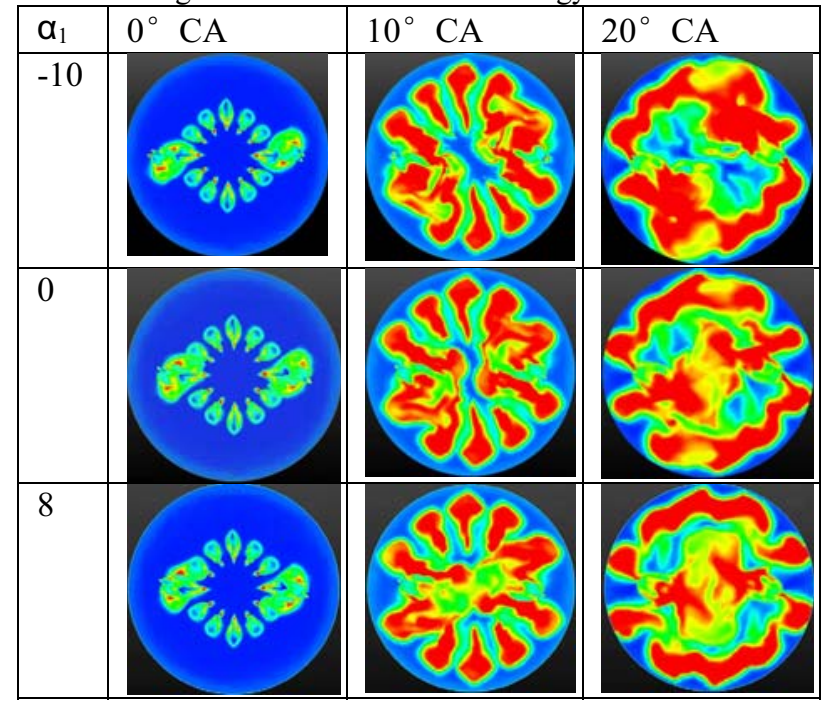




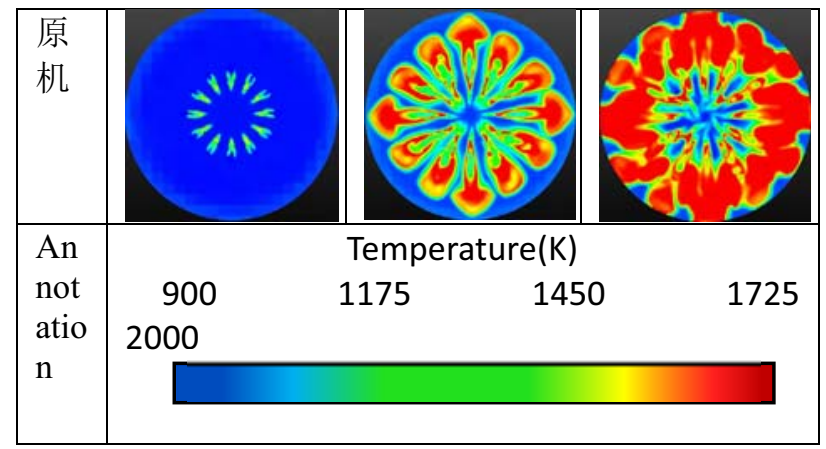

Figure 5. Influence of $\alpha_{1}$ on temperature field distribution

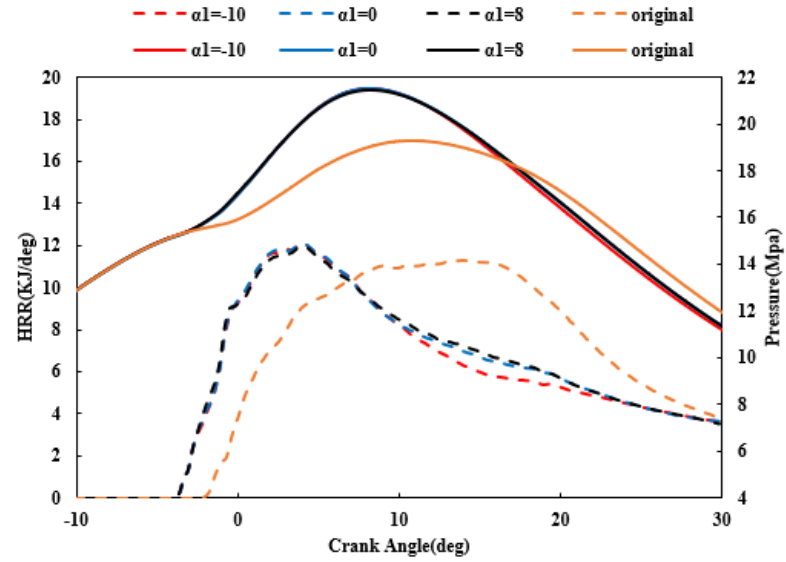

Figure 6 Influence of $\alpha_{1}$ on HR-Rate

The influence of injection orientation angle $\left(\alpha_{2}\right)$ on combustion performance is shown in figure 7,8 and 9 . Considering the wall impingement and the jet-jet interaction of the fuel spray of side injector, the variation range of $\alpha_{2}$ is set between 19 and 23 degree. As $\alpha_{2}$ increases, the heat loss decreases and exhaust loss increases. The later energy loss indicates a dominated influence on the effective thermal efficiency. When the $\alpha_{2}$ increases, the distribution of high temperature region becomes more dispersed (shown in Figure8). This is because the increased interaction between fuel jets from side injector and central injector, which is indicated by the less penetration of fuel spray from side injector with bigger $\alpha 2$ shown in figure 9 . It leads to a worse fuel/air mixture and a decrease in the heat release rate at the later stage of combustion (shown in figure 9). In addition, the reduction of high temperature region near cylinder wall as $\alpha 2$ increase might be responsible for the reduction in heat loss. Overall, the reduction in $\alpha 2$ leads to reduced fuel jets interaction and better utilization of air in the combustion chamber and consequently increases the effective thermal efficiency.

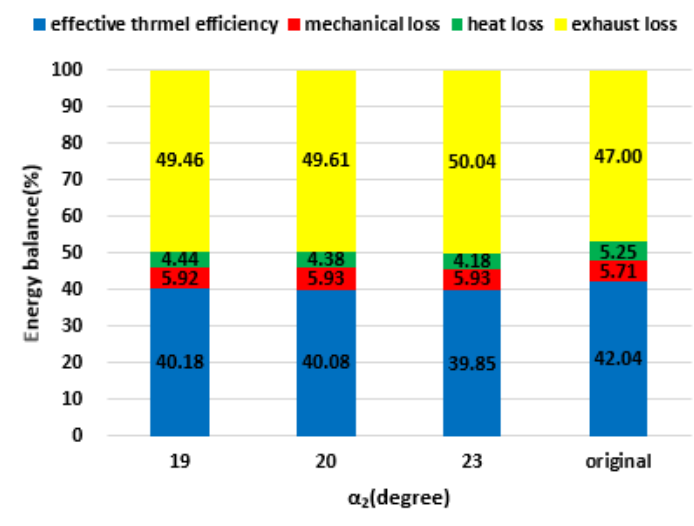

Figure. 7 Influence of $\alpha_{2}$ on energy balance

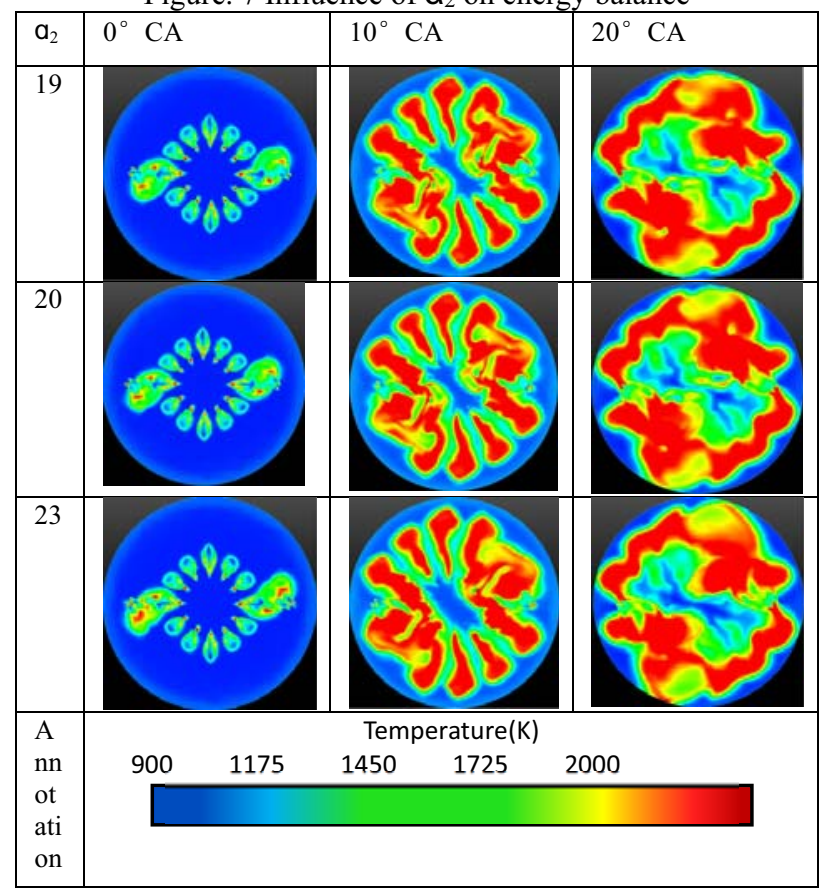

Figure 8. Influence of $\boldsymbol{\alpha}_{2}$ on temperature field distribution

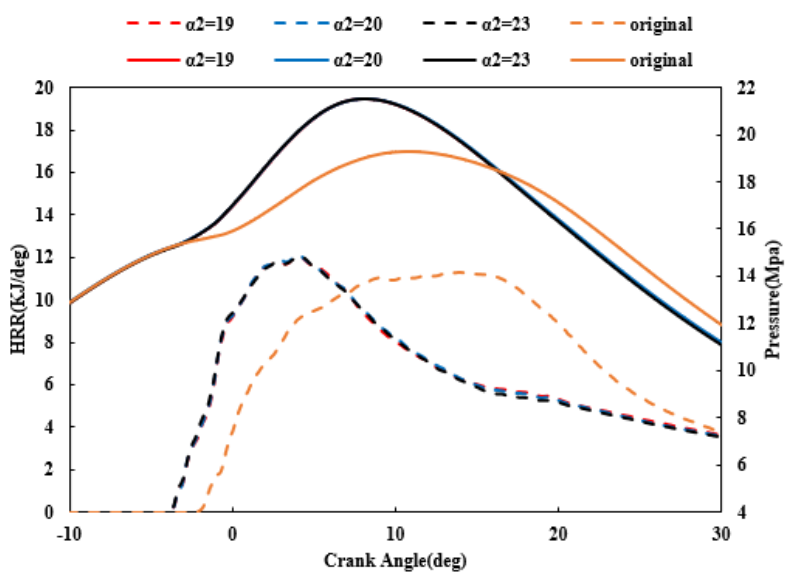

Figure 9 Influence of $\boldsymbol{\alpha}_{2}$ on HR-Rate

\subsection{Influence of longitudinal section Angle}

Similarly, the influence of the longitudinal section angle (LA) on the combustion and performance is shown in figure 10, 11 and. From the energy balance and heat release rate analysis in figure 10 and 11, the effect of angle 
$\beta 1$ is slight, the difference is less than $0.03 \%$ for thermal efficiency. There is no obvious difference for other characteristics, such as heat and exhaust loss. For the other angle $\beta 2$, there is only a slightly improvement in heat loss and thermal efficiency when $\beta 2$ decreases from 12.5 to 10.5 degree although its exhaust loss increased slightly. As $\beta 2$ further increases to 14.5 degree, an
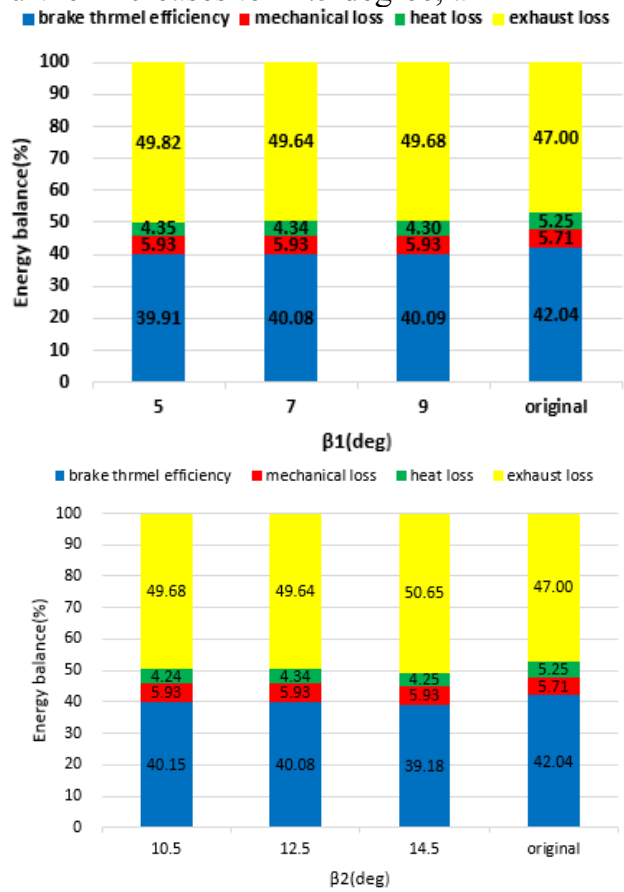

Figure.10 Influence of $\beta_{1}$ and $\beta_{2}$ on energy balance

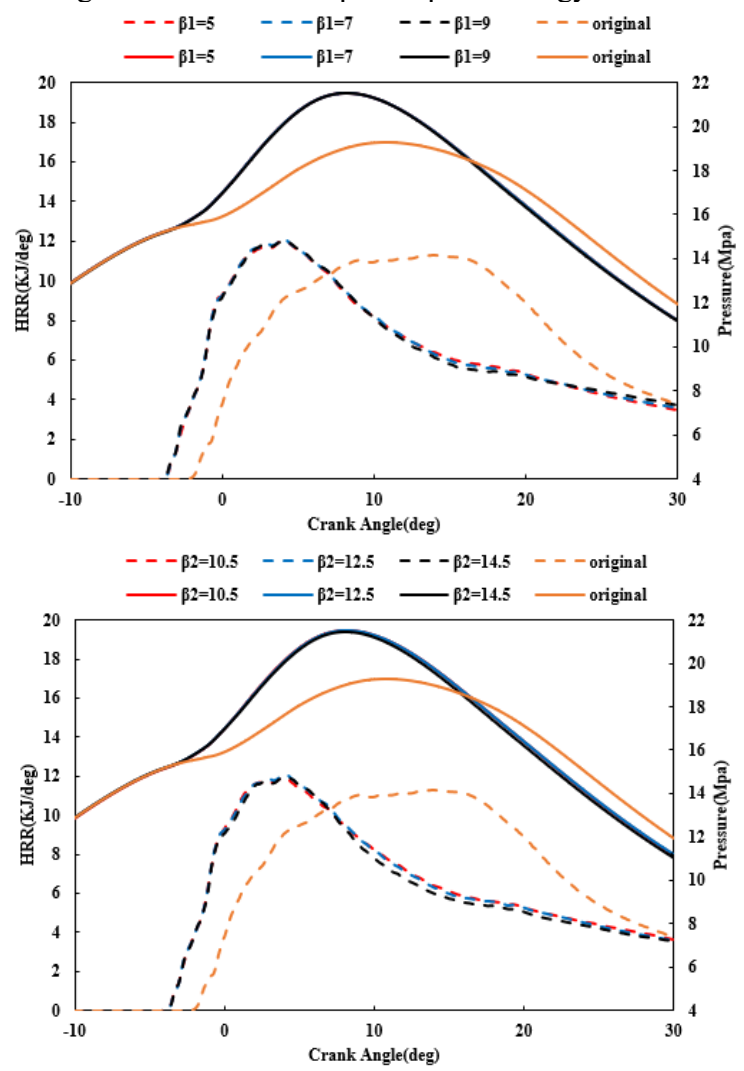

Figure 11 Influence of LA on HR-Rate obvious reduction of thermal efficiency and heat release rate can be observed in figure 10and 11. This might be because the larger angle of lower spray orientation increases the impingement of cylinder head at central part and consequently deteriorates the combustion and increases the exhaust loss. Overall, the longitudinal section angle can be confirmed as 7 and 12,5 degree for further optimization.

\subsection{Impact of fuel injection strategy}

As mentioned above, optimization of the injection angle can improve the effective thermal efficiency. However, the thermal efficiency is still lower than that of original engine, due to the smaller fuel flow the side injector, which limited by the space of cylinder head and specification of fuel injectors. If the nozzle hole diameter of side injector can be modified to achieve a higher flow rate, the thermal efficiency has the potential to be further improved ${ }^{[11,12]}$. In addition, the previous fuel injection quantity of the side injector leads to longer injection and combustion duration significantly compared with those of original engine, which is the main reason for the low thermal efficiency in previous cases. This

section aims to solve the two problems by optimizing the fuel injection strategy and hole diameter based on the optimized injection orientation angle in previous section. The simulation cases are shown in Table 4.

Figure 12, 13, and 14 compare the effects of injection strategies on combustion characteristics and energy balance. It can be seen that increasing the fuel injection quantity ratio of the main injector (case 1,2 and 3) is beneficial to increase the effective thermal efficiency. This is due to the fact that the overall injection duration is reduced as the fuel ratio reduced from 4:3:3 to $4: 1: 1$ with the same fuel injection quantity. Thus, the combustion is improved dramatically (shown in figure 13) and consequently increases the heat loss slightly and decre ases the exhaust loss obviously. It should be noted that the heat loss of case 3 is slightly lower than that of case 2 , and the increase of effective thermal efficiency is not large. Comparing the temperature distribution of case 1,2 and 3 in figure 14 , it is obvious that the high-temperature zone in the combustion chamber tends to be more uniformly distributed, which means that the fuel/air mixture is improved and it is also confirmed by the results of heat releases rate at later combustion period in figure 13. As can be seen from figure 13, the heat release rate of case 3 after 25ATDC is lower than case 2 . This change means that the temperature in the Figure 13. Influence of fuel injection strategy on HR-Rate and in-cylinder pressure combustion chamber of case 3 later is lower than 
Table 4, Simulation cases matrix

\begin{tabular}{|c|c|c|c|c|c|c|}
\hline case & $\begin{array}{l}\text { Distribution ratio } \\
\text { of injection } \\
\text { volume }\end{array}$ & $\begin{array}{l}\text { Main spray start } \\
\text { time (CA) }\end{array}$ & $\begin{array}{l}\text { Start of side spray } \\
\text { (CA) }\end{array}$ & $\begin{array}{l}\text { Main injector } \\
\text { duration } \\
\text { (deg) }\end{array}$ & $\begin{array}{l}\text { Side injector } \\
\text { duration } \\
(\operatorname{deg})\end{array}$ & $\begin{array}{l}\text { Dimensions of } \\
\text { side injector } \\
\text { orifice }(\mathrm{mm})\end{array}$ \\
\hline 1 & $4: 3: 3$ & -5 & -5 & 11.265 & 25.948 & 0.63 \\
\hline 2 & $2: 1: 1$ & -5 & -5 & 12.917 & 21.655 & 0.63 \\
\hline 3 & $4: 1: 1$ & -5 & -5 & 15.761 & 15.728 & 0.63 \\
\hline 4 & $2: 1: 1$ & -5 & -5 & 12.917 & 14.181 & 0.8 \\
\hline 5 & $2: 1: 1$ & -7 & -5 & 12.917 & 14.181 & 0.8 \\
\hline
\end{tabular}

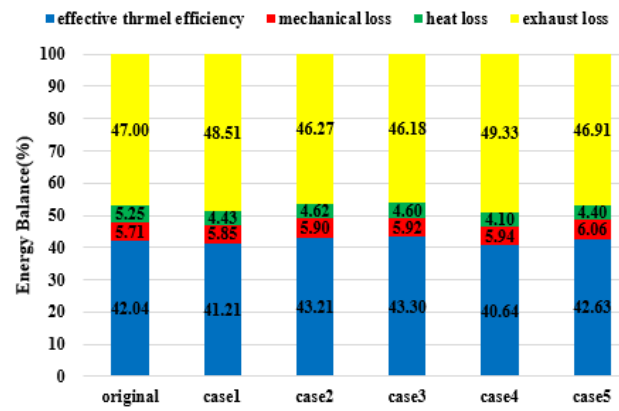

Figure 12 Influence of fuel injection strategy on energy balance
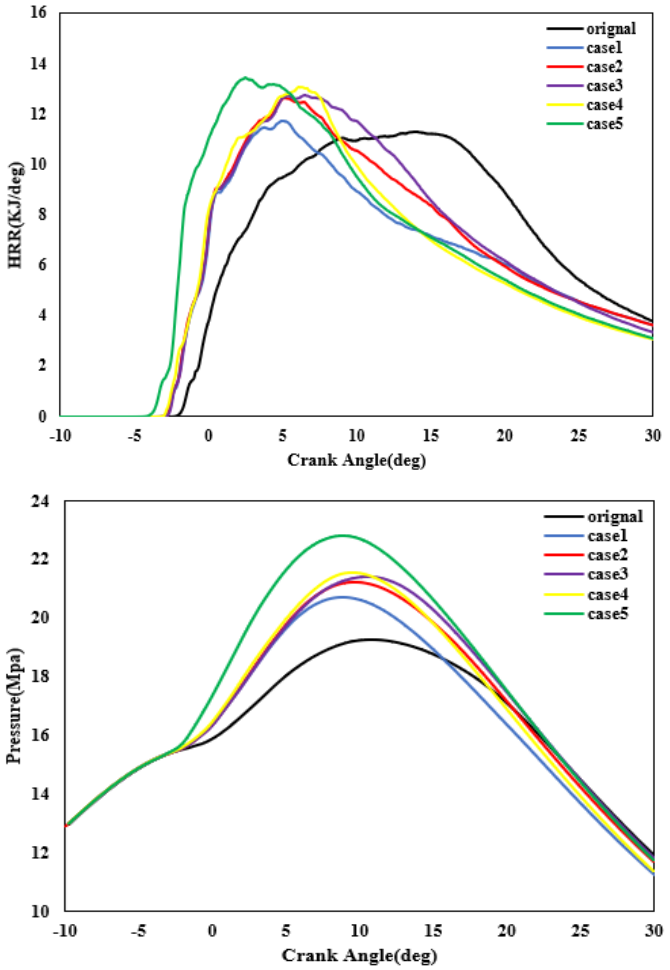

Figure 13. Influence of fuel injection strategy on HR-

Rate and in-cylinder pressure

case 2. This trend explains that the heat loss of case 3 is slightly lower than case2. As can be seen from Figure 15, although the combustion

\begin{tabular}{|l|l|l|l|}
\hline Case & $0^{\circ} \mathrm{CA}$ & $10^{\circ} \mathrm{CA}$ & $20^{\circ} \mathrm{CA}$ \\
\hline
\end{tabular}

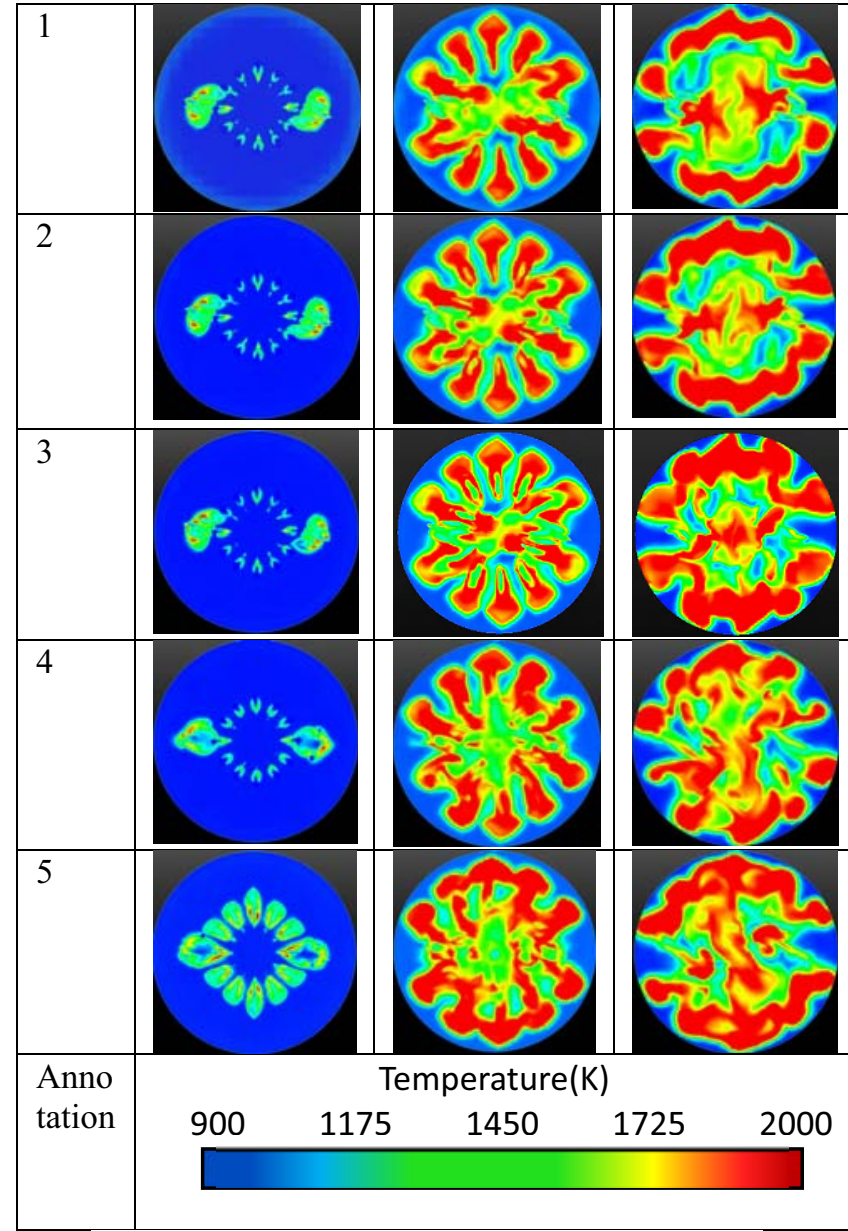

Figure 14. Influence of strategy on temperature field distribution

temperature is increased as the heat release increased, the brake specific NOx emissions of case 1,2 and 3 are still less than that of original engine, because brake power output is improved as fuel quantity ratio increased. It is also the reason to explain the significant reduction of the exhaust loss while the in-cylinder pressure and temperature is increased. By maintaining the fuel quantity ratio as 2:1:1 and injection timing with increasing the nozzle hole diameter of side injector, further simulation is conducted in case 4 and compares with case 2 . Although the fuel flow of side injector is increased, the in-cylinder combustion was not improved due to the impact of worse the fuel breakup and atomization for the injector of larger 
nozzle hole. An obvious drop can be observed at diffusion combustion period in the heat release rate for case 4 . Comparing the temperature distribution of case 4 to that of case 2, the combustion of side injection fuel is not obviously observed at 10 CAD ATDC. The incomplete combustion at case 4 leads to increasing of exhaust loss dramatically and the reduction of in-cylinder the heat loss due to the lower combustion temperature. However, the benefit of heat loss still can't compensate the exhaust lo ss and results in a low thermal efficiency. By further advancing the injection time of the main injector from 5CAD to -7CAD, the effective thermal efficiency is which is indicated by the heat release rate in figure 13 . However, the combustion of fuel injection of side injector is still not obvious observed based on the temperature distribution in figure 14, which leads to an increased in case 5 . This is due to the advance of the injection angle leads to an earlier start and end of combustion, unchanged heat release rate in the later stage of combustion shown in figure 13. However, the advanced injection timing also increased the heat loss, mechanical loss and NOx emission, because it increased the combustion peak pressure, which reduces the effect of thermal efficiency improvement. Overall, decreasing the injection duration might be the main factor to improve thermal efficiency and NOx emission at the same time.

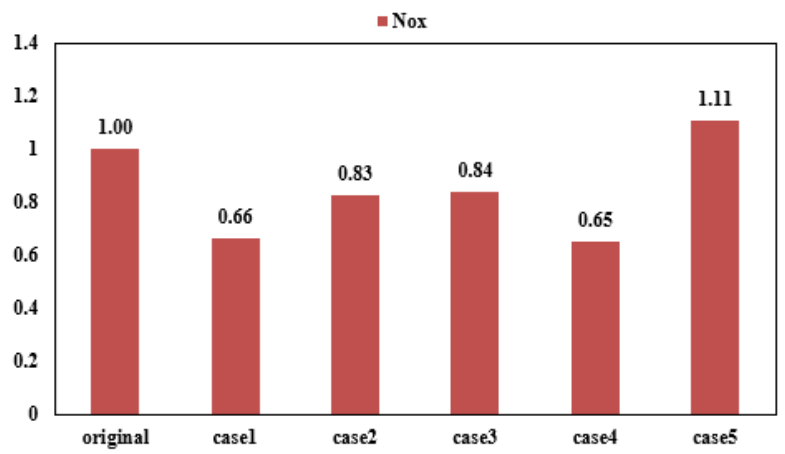

Figure 15. influence of strategy on emission characteristic

\section{Conclusion}

In order to investigate the influence of fuel injection direction and strategy on engine thermal efficiency and emission, the multi-injector system is developed in a CFD large bore marine medium speed engine. Comparing the results of multi-injector system to those of the original engine, the main conclusions are as follows:

- Heat loss is always reduced by applying the multiinjector system because part of fuel is combusted at the center of combustion chamber and consequently reduced near wall combustion.

- Compared with the injection direction of longitude, the influence of the section injection direction on the energy balance is more obvious. The appropriate selection of section Angle can improve the thermal efficiency. It should be noted that $\beta_{2}$ needs to be carefully designed to avoid oil and gas hitting the cylinder head. Otherwise, the thermal efficiency would be reduced obviously.

- By adjusting the ratio of the amount of oil between three injectors, shorten the injection duration, can significantly improve the thermal efficiency effectively. When the total injection time of the three injectors is the shortest, the thermal efficiency can be improved by 1.26

$\%$ and the NOx can be reduced by $16 \%$.

- Increasing the nozzle hole diameter of the side injector does not shorten the overall duration of combustion, but decreases the effective thermal efficiency due to the deteriorate fuel atomization and evaporation.

- Advanced injection timing of central injector increases the thermal efficiency obviously, but can't improve other characteristics, such as NOx emission, heat loss, exhaust loss, peak in-cylinder pressure and maximum pressure rise rate. Other strategies should be combined to avoid those side effects of using advanced injection timing.

- Compared with the single injector, the distribution of temperature field in the combustion chamber is more uniform when using multiple injectors, and the high temperature area in the center of the combustion chamber increases, effectively increases the air utilization rate and reduces heat release.

\section{Acknowledgments}

The authors gratefully acknowledge the support of Research of Controllable Combustion Technology in Marine Diesel Engine (granted No. 2017YFE0116400) from National Key R\&D Program of China, Ministry of Science and Technology.

\section{Reference}

1. Koci, C., Florea, R., Das, S., Walls, M., Simescu, S., Roberts, C. (2013) Air-assisted direct injection diesel investigations. SAE technical paper 2013-01-0907.

2. Nakagome, K., Shimazaki, N., Niimura, K., Kobayashi, S.(1997) Combustion and emission characteristics of premixed lean diesel combustion engine. SAE technical paper 970898.

3. Hashizume, T., Miyamoto, T., Hisashi, A., Tsujimura, K., (1998) Combustion and emission characteristics of premixed lean diesel combustion engine. SAE technical paper 980505.

4. Nyrenstedt,G., Al Turkestani,T., Im, H., Johansson, B.,

(2019)"CFD Study of Heat Transfer Reduction Using Multiple Injectors in a DCEE concept". SAE technical Paper 2019-01-0070.

5. Nyrenstedt, G., Im,H ., Andersson, A., Johansson, B., (2019) "Novel Geometry Reaching High Efficiency for Multiple Injector Concepts," SAE technical Paper 2019-01-0246. 
6. Noboru, U., Takeshi, O., Hiroki, W., (2018) A new concept of actively controlled rate of diesel combustion for improving brake thermal efficiency of diesel engines: Part I - verification of the concept. International J of Engine Research Vol. 19(4) 474487.

7. Noboru, U., Hiroki, W., (2019) A new concept of actively controlled rate of diesel combustion (ACCORDIC): Part II-simultaneous improvements in brake thermal efficiency and heat loss with modified nozzles. International J of Engine Research, Vol. 20(1) 34-45

8. Takeda, Y., Niimura, K., Bingquan, Z., (1998) Combus-tion and emission characteristics of diesel engines using multiple injector systems. Foreign internal combustion engine ,4(2): 19-28.

9. Nyrenstedt, G., Watanabe, K., Enya, K., Shi, H., Uchida, N., Johansson, B., (2019) Thermal Efficiency Compar-ison of Different Injector Constellations in a CI Engine. SAE International Technical Paper.201924-0172,

10. Beat, R., Kai, H., Konstantinos, B., (2015) Experimental investigation on the characteristics of sprays represent-tative for large 2-stroke marine diesel engine combustion systems. SAE International Paper,2015-01-1825.

11. Graf, G., Seitz, H., Theissl, H., Machold, A., (2017) Further ther mal efficiency increase of the diesel combustion for com mercial engines. In: Proceedings of 4th ATZ international engine congress, Baden.212-223.

12. Kocher,L.,(2016) Cummins 55\% BTE project, presentation material in 2016 US DOE Annual Merit
Review,2016,https://www.energy.gov/sites/prod/files /2016/06/f32/ace098_kocher_2016_o_web.pdf. 